http://dx.doi.org/10.35381/e.k.v3i6.818

\title{
Recursos tecnológicos empleados en infocentros por facilitadores de las provincias de Azuay y Cañar
}

\section{Technological resources used in info centers by facilitators from the provinces of Azuay and Cañar}

\author{
Blanca Azucena González-Andrade \\ blanca.gonzalez.84@est.ucacue.edu.ec \\ Universidad Católica de Cuenca, Azogues \\ Ecuador \\ https://orcid.org/0000-0003-1705-9300 \\ Darwin Gabriel García-Herrera \\ dggarciah@ucacue.edu.ec \\ Universidad Católica de Cuenca, Azogues \\ Ecuador \\ https://orcid.org/0000-0001-6813-8100 \\ Luis Bolívar Cabrera-Berrezueta \\ bolivarcabrera@ucacue.edu.ec \\ Universidad Católica de Cuenca, Cuenca \\ Ecuador \\ https://orcid.org/0000-0002-6853-635X \\ Juan Carlos Erazo-Álvarez \\ jcerazo@ucacue.edu.ec \\ Universidad Católica de Cuenca, Cuenca \\ Ecuador \\ https://orcid.org/0000-0001-6480-2270
}

Recepción: 09 abril 2020

Revisado: 15 de mayo 2020

Aprobación: 15 junio 2020

Publicación: 1 de julio 2020 


\title{
RESUMEN
}

La investigación tiene su objetivo en analizar el uso y aplicación de los recursos tecnológicos por parte de los facilitadores de los infocentros de las provincias de Azuay y Cañar. Siendo de carácter descriptivo correlacional no experimental transeccional. Se revela una intima relación entre las variables de uso de presentaciones mediante powtoon y clases impartidas mediante plataformas como: Zoom, Classroom, Facebook live, entre otras, así se puede observar que dichos recursos tecnológicos despiertan el interés en los facilitadores para su posible aplicación dentro de estos espacios comunitarios llamados infocentros. Evidenciándose la estrecha relación que existe entre la aplicación y predisposición por parte de un $80 \%$ de facilitadores para aplicar nuevos recursos tecnológicos.

Descriptores: Enseñanza audiovisual; enseñanza multimedia; tecnología de la información; gobierno electrónico. (Palabras tomadas del Tesauro UNESCO).

\begin{abstract}
The research aims to analyze the use and application of technological resources by the facilitators of the infocentres in the provinces of Azuay and Cañar. Being descriptive correlational non-experimental transectional. An intimate relationship is revealed between the variables of use of presentations through powtoon and classes taught through platforms such as: Zoom, Classroom, Facebook live, among others, thus it can be seen that said technological resources arouse interest in the facilitators for their possible application within of these community spaces called infocentres. Evidencing the close relationship that exists between the application and predisposition on the part of $80 \%$ of facilitators to apply new technological resources.
\end{abstract}

Descriptors: Audiovisual instruction; multimedia instruction; information technology; online governance. (Words taken from the UNESCO Thesaurus). 


\section{INTRODUCCIÓN}

Las TIC han transformado drásticamente la dinámica del desarrollo a nivel mundial puesto que hoy en día existen programas y proyectos dedicados exclusivamente a romper la famosa brecha digital. Tal como lo afirma (Raymond, 2008) los países especialmente subdesarrollados y sus zonas particularmente aisladas cuentan en la actualidad con la oportunidad de reducir las barreras geográficas que históricamente les han impedido intercambiar conocimiento e información con el resto del mundo.

En nuestro país Ecuador el Ministerio de Telecomunicaciones y de la Sociedad de la Información [MINTEL] ha implementado infocentros comunitarios a nivel nacional mismos que son espacios destinados a garantizar el acceso gratuito a las TIC, realizar capacitaciones en temas tecnológicos, emprendimientos y servicios electrónicos de internet con la finalidad de eliminar la brecha digital en los puntos más vulnerables del país que no cuentan con acceso a los recursos tecnológicos y mucho menos a internet, por lo que los facilitadores pretenden alcanzar su meta al capacitar a la mayor cantidad posible de personas que se encuentran en las zonas vulnerables y de difícil acceso tecnológico.

En pleno siglo XXI todavía existen falencias con el funcionamiento y manejo de las tecnologías tal como lo describe (Cabero Almenara, 2015) las Tecnologías de la Información y Comunicación [TIC], Tecnologías del empoderamiento y la participación [TEP], y Tecnologías para el Aprendizaje y el Conocimiento [TAC] son herramientas digitales que no son aprovechadas como lo deberían de ser entre los problemas más relevantes radica en que aún existen comunidades que no tienen acceso a los recursos tecnológicos lo que provoca un atraso académico ya que nos encontramos alejados del conocimiento y uso de tecnologías que hoy en día son indispensables para la comunicación, información, educación, agilización de trámites, entre otros.

(Delgado, Wardlow, Mcknight, \& O’Mal, 2015) afirman que la integración de la tecnología ha cambiado en todo el mundo pues a medida que iniciamos en la era digital, es de suma importancia equipar a los estudiantes con tecnología, entonces los maestros y 
administradores son quienes deben buscar los mejores métodos para integrar las TIC en el currículo y en las instituciones educativas, siendo una alternativa comprar los instrumentos tecnológicos necesarios, más sin embargo es evidente el importante déficit presupuestario por lo que no sería factible para todas las instituciones, como tal hay un amplio espectro de tecnología entornos que pueden ofrecer contenidos educativos facilitados directamente por internet ya sea completamente en línea o a través del aprendizaje combinado.

Así mismo lo manifiestan (Jama Zambrano \& Cornejo Zambrano, 2016) los sistemas educativos en todo el mundo afrontan el reto de utilizar correctamente las nuevas tecnologías para proveer a sus estudiantes con las herramientas y conocimientos necesarios para prepararlos para la nueva era digital del siglo XXI, sin embargo, la falta de conocimiento es notable, por cuanto existe una gran diferencia en el uso y manejo de las tecnologías ya sea por edad, sexo o región, para aportar con el aprendizaje y uso correcto de los recursos tecnológicos mediante el uso de las TIC, es más notorio aún la ausencia de estos recursos y conocimientos en las comunidades rurales y urbano marginales del país (Siguenza-Peñafiel, Erazo-Álvarez, \& Narváez-Zurita, 2020).

En Ecuador la constitución en el artículo 347 menciona que el estado "incorporará las tecnologías de la información y comunicación en el proceso educativo y propiciará el enlace de la enseñanza con las actividades productivas o sociales (Asamblea Nacional, 2008) lo que ha provocado que en la actualidad la educación de un giro irrevocable, incitando a dejar atrás la educación tradicionalista, para centrarnos en el tema de inclusión de tecnologías en la educación para facilitar el aprendizaje y acceso a las mismas en todas las regiones del país (Picón-Vizhñay, Erazo-Álvarez, \& Narváez-Zurita, 2019).

Además la ley Orgánica de Telecomunicaciones (Asamblea Nacional, 2015), en su artículo 88 indica que el Ministerio rector de las Telecomunicaciones deberá promover la sociedad de la información y del conocimiento para el desarrollo integral del país, de tal manera que garantice el derecho a la comunicación y el total acceso a la información 
puesto que es el ente encargado de promover el acceso universal a los servicios de telecomunicaciones de manera especial, en zonas rurales o urbano marginales, a fin de asegurar estos servicios en beneficio de los ciudadanos ecuatorianos.

\section{Las TIC en la actualidad}

Mientras que el mundo está digitalizando la comunicación y sus métodos de coordinación en diferentes lugares de la sociedad se les presenta a los países más atrasados la oportunidad de alcanzar al resto, mientras tanto que los países en desarrollo pueden avanzar extraordinariamente en la actualización de la estructura institucional existe a través de la digitalización en los distintos sectores electrónicos.

Esto significa que el conocimiento puede ser compartido por las Tecnologías de la Información y Comunicación [TIC], ya que contribuyen al cambio de una sociedad de modo fundamental en el contexto de desarrollo y evolución educativa. (Cabero Almenara, 2015) hace un llamado a la reflexión sobre el uso y aplicaciones correctas de las tecnologías para trasformar la practica educativa. Pues los procesos de aprendizaje que son apoyados por entornos virtuales radican en las herramientas con las que cuentan los docentes para formar ciudadanos que sean participativos y profesionales de calidad, más sin embargo, se tiende a confundir las herramientas tecnológicas con la supuesta libre circulación de la información, algo que radicalmente no siempre es cierto, por cuanto las personas suelen acercarse a los bloques informativos afines a su entendimiento o incluso a su sola atracción visual, esto debido a su carente competencia para aprender a utilizar todas las herramientas digitales que se nos presentan en la actualidad.

(España-Chavarria \& Canales-Garcia, 2013) explican que las tecnologías no son únicamente de la información y comunicación, son también del conocimiento para la vida. De tal manera que radica la importancia de estos recursos en la educación y crecimiento diario debido a la adquisición de nuevos conocimientos mediante varias aplicaciones con el solo hecho de poder contar con un computador que tenga conexión a internet las 
personas se encuentran con la oportunidad de realizar cualquier tipo de investigación, trabajos educativos, comunicaciones inmediatas con los demás, por tanto se presenta la necesidad de aprender a manejar correctamente las tecnologías para poder utilizarlas en el día a día.

Pues su vinculación con la educación permite tener diferentes visiones ya que por una parte la concepción la concepción que en la actualidad se posee del aprendizaje es obviamente diferente a la que imperaba en la antigua sociedad donde no se contaba con los recursos tecnológicos necesarios y casi indispensables hoy en día, de manera que se enfrentaba a la concepción del aprendizaje como memorización de la información, muy diferente a lo que hoy buscamos con un verdadero aprendizaje donde nos centramos en el estudiante donde se realiza una verdadera conexión, proceso y re-estructuración de la información.

Pues según (Poloni, 2019) la tecnología de la información, ha cambiado principalmente la forma en que se usa y produce el contenido, pues en la medida que la tecnología va avanzando también han ido revolucionando las dinámicas sociales, y culturales de manera que mediante el uso de las TIC se logra obtener mayor atención a esta revolución digital que puede ayudar a hacer la información más atractiva para los usuarios. Por otro lado, (Vargas Espin \& Parra Balza, 2018), en su investigación nos pone en manifiesto que el Gobierno del Ecuador a través del Ministerio de Telecomunicaciones y de la Sociedad de la Información ha implementado espacios de participación ciudadana llamados Infocentros comunitarios, donde las persona pueden acudir a realizar sus tareas, a capacitarse, realizar actividades que requieran de herramientas gubernamentales en línea, entre otras, todo esto de manera gratuita con la finalidad de romper la brecha digital de acceso a las herramientas tecnológicas y el analfabetismo digital en Ecuador, sin embargo al parecer no todas las personas pueden acudir a estos centros para educarse continuamente, por ello es recomendable que tomaran cursos de capacitaciones cada cierto tiempo, puesto que están a su alcance durante todo el año. 


\section{Uso de Recursos Tecnológicos en Infocentros}

Según (Jama Zambrano \& Cornejo Zambrano, 2016) la formación docente en los últimos años ha sufrido una transformación en proporción a los contenidos, orientaciones y medios. Puesto que el desarrollo de nuevos recursos didácticos y tecnologías educativas han provocado que los docentes que participan en los esfuerzos de formación estudiantil y capacitación adquieren un mayor protagonismo intervención y control de los procesos educativos más aún al hacer uso de los recursos y herramientas que mejor se adaptan a sus necesidades formativas, de tal manera que los estudiantes puedan desarrollar la capacidad de entendimiento y que mejor aún que logren realizar un aprendizaje combinado tanto teórico como práctico (Serrano-de-Barrios, 2019).

De tal manera que la implementación de las Tecnologías de la Información y Comunicación [TIC] dentro de la sociedad del conocimiento ha provocado varios cambios en el proceso de la información, mediante el uso de computadores y sus programas que nos permiten establecer, modificar, almacenar y salvaguardar dicha información lo que conlleva a aprovechar las ventajas de estos recursos tecnológicos que permiten innovar la educación y aportan a la creación y desarrollo de nuevos conocimientos, como lo asevera (Hernandez, 2017),

De tal manera que este proceso de incorporación de las [TIC] en educación va más allá de las herramientas tecnológicas puesto que se dice de una construcción didáctica para consolidar un aprendizaje significativo mediante el uso de las tecnologías, de tal manera que se debería implementar las Tecnologías de la Información y Comunicación [TIC], Tecnologías del empoderamiento y la participación [TEP], y las Tecnologías para el Aprendizaje y el Conocimiento [TAC] en el proceso de aprendizaje para facilitar a las personas que se acercan a los centros educativos su uso y manejo incorporando estrategias que optimizan el proceso de aprendizaje.

Hasta el año 2016 que es donde se realizó la última actualización de datos en Ecuador se cuenta con 886 infocentros según las estadísticas del Sistema Nacional de Información [SNI], en la actualidad se estima que son 890 infocentros que constan el 
Ministerio de Telecomunicaciones [MINTEL] siendo espacios comunitarios destinados a brindar internet gratuito, capacitaciones, servicios en línea y acompañamiento en emprendimientos a las personas que se encuentran en zonas de difícil acceso a las tecnologías y a internet se encuentran ubicados específicamente en las comunidades rurales y urbano marginales del país.

Es así que (Calderon \& Acuña, 2017) manifiestan que el acceso público a las TIC a través de los infocentros ha transformado las iniciativas ciudadanas ya que se ha empoderado de estas herramientas necesarias a los sectores marginales de la población y ha fortalecido a los proyectos del gobierno central pues en el área de las Telecomunicaciones todos los sectores vulnerables cuentan no solo con la dotación de equipamiento y conectividad sino también con capacitaciones permanentes sobre el buen uso de las TIC, bajo el indicio de que el acceso a Internet y a las telecomunicaciones debería ser un bien público, el Ministerio de Telecomunicaciones pretende universalizar las TIC a través de los planes y programas dentro de los infocentros comunitarios como son: Conectividad Escolar, Aulas Móviles, Capacitaciones del Plan de Alistamiento Digital [PLANADI] entre otros, bajo el marco de la Estrategia Ecuador Digital 2.0.

Para el año 2012, se construyeron, capacitaron y equiparon a 373 Infocentros, se espera llegar a 820, en la totalidad de las juntas parroquiales a nivel nacional o incluso superar este número con la finalidad de democratizar a las TIC y fomentar el conocimiento y uso de las mismas para disminuir la llamada brecha digital de manera que se pueda promover el uso de los servicios gubernamentales en línea y motivar a las creación de proyectos y soluciones tecnológicas. 
Revista Electrónica de Ciencias de la Educación, Humanidades, Artes y Bellas Artes

Año III. Vol III. Nº6. Julio - Diciembre 2020

Hecho el depósito de Ley: FA2018000022

ISSN: 2665-0282

FUNDACIÓN KOINONIA (F.K)

Santa Ana de Coro, Venezuela

Blanca Azucena González-Andrade; Darwin Gabriel García-Herrera; Luis Bolívar Cabrera-Berrezueta; Juan Carlos Erazo-Álvarez
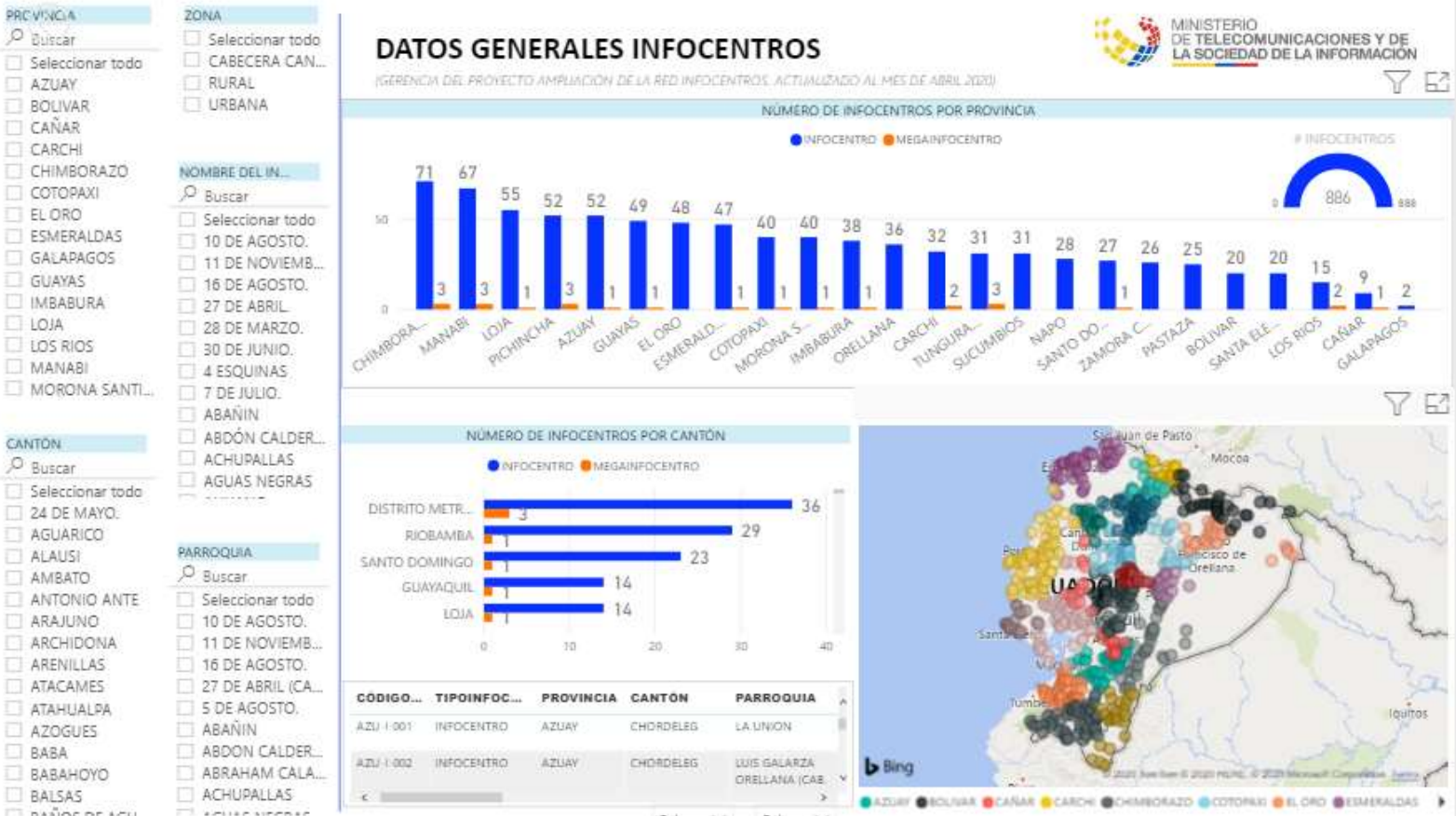

Figura 1. Estadísticas de Infocentros a nivel Nacional según el SNI Fuente: (MINTEL, 2016).

En tal sentido la presente investigación se centra en analizar la aplicación de los recursos tecnológicos por parte de los facilitadores de los infocentros comunitarios de las provincias de Azuay y Cañar denotando la importancia del uso correcto de las tecnologías dentro de estos espacios comunitarios, para poder proponer estrategias que fortalezcan el uso correcto de nuevos recursos tecnológicos para mejorar el proceso de aprendizaje por parte de los usuarios de dicha entidad pública, pues la utilización de estas herramientas cada vez aumentan en nuestras instituciones educativas, pero nos preguntamos qué sucede con las personas que están en lugares que aún no tienen acceso a estos recursos, por ello el papel fundamental de los infocentros es llegar hasta los puntos más vulnerables que no cuentan con acceso a las herramientas tecnológicas y mucho menos a internet. Acogida de los infocentros a nivel nacional entre visitas y capacitaciones según las estadísticas del SNI tenemos: 
Revista Electrónica de Ciencias de la Educación, Humanidades, Artes y Bellas Artes

Año III. Vol III. Nº6. Julio - Diciembre 2020

Hecho el depósito de Ley: FA2018000022 ISSN: $2665-0282$

FUNDACIÓN KOINONIA (F.K)

Santa Ana de Coro, Venezuela

Blanca Azucena González-Andrade; Darwin Gabriel García-Herrera; Luis Bolívar Cabrera-Berrezueta; Juan Carlos Erazo-Álvarez

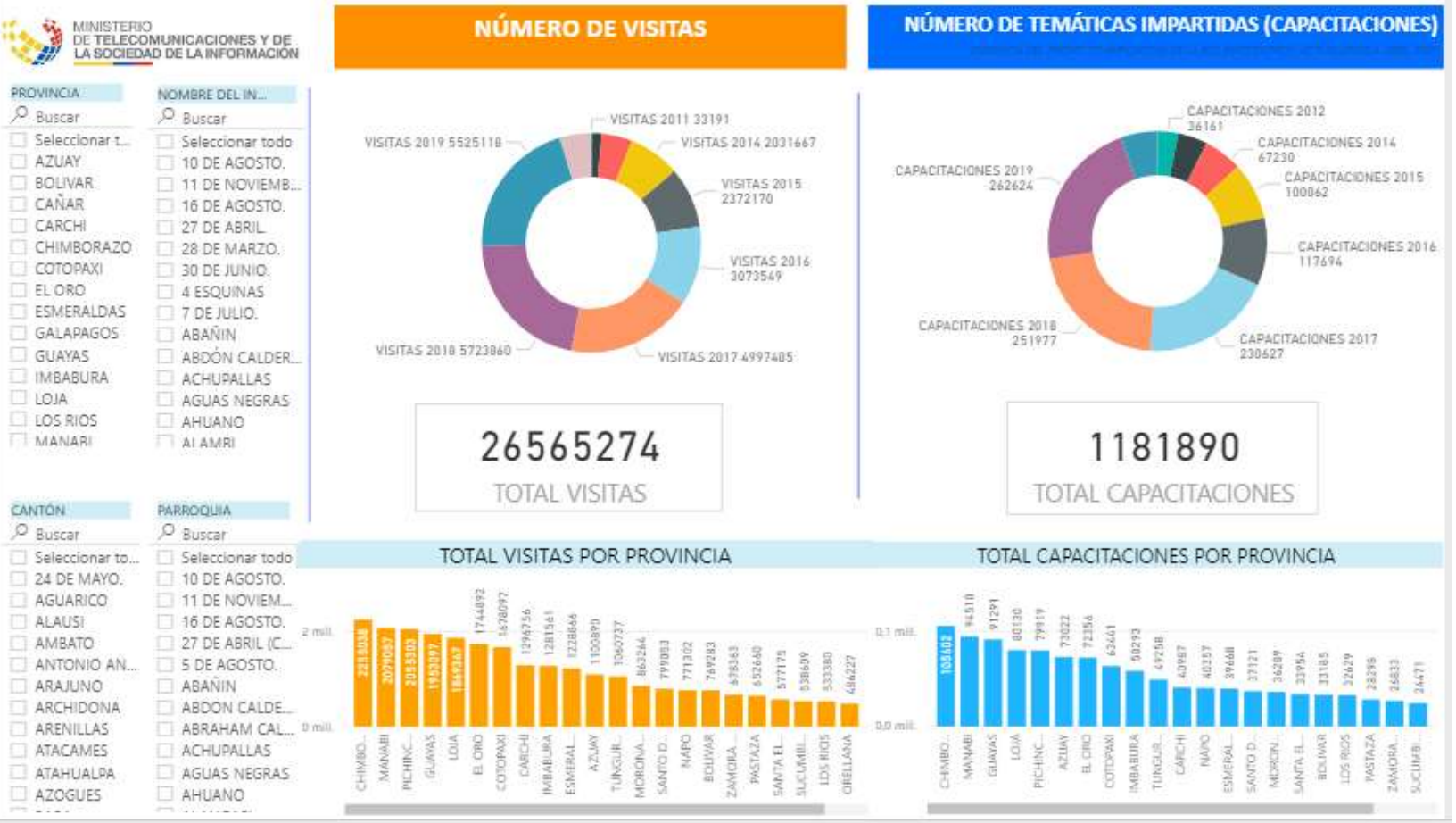

Figura 2. Estadísticas de Infocentros a nivel Nacional según el SNI Fuente: (MINTEL, 2016).

Claro está que el uso de los recursos tecnológicos provoca cambios indiscutiblemente en la educación, de manera tal que los infocentros son entes que tratan de incorporar en la manera de lo posible el uso correcto de las TIC, en la actualidad radica la importancia del aprendizaje y manejo de estas tecnologías para poder agilizar el aprendizaje y mantenernos comunicados e informados mediante los aparatos electrónicos con lo que contamos los ciudadanos (Arroyo-Carrera, et al., 2020).

De ésta manera la finalidad de la investigación tiene su objetivo en analizar el uso y aplicación de los recursos tecnológicos por parte de los facilitadores de los infocentros de las provincias de Azuay y Cañar. 


\section{MÉTODO}

La presente investigación es de carácter descriptivo correlacional no experimental transeccional, por lo que se recopiló información en único momento, sin manipulación deliberada de las variables por parte de los investigadores (Pereira-Perez, 2011), la población estuvo conformada por 62 personas que se encuentran a cargo de los infocentros de las diferentes áreas rurales y urbano marginales de las provincias de Azuay y Cañar del Ecuador (López-Intriago, Erazo-Álvarez, Narváez-Zurita, \& Moreno, 2020).

Para la recopilación de la información se empleó la encuesta como técnica de modo online, mediante un instrumento tipo cuestionario en escalamiento Likert con un confiabilidad de Alfa de Cronbach de 0,92 de ese modo, lo datos recopilados fueron procesados estadisticamente mediante analisis descriptivo y Chi-cuadrado de Pearson (Martín, 2011). 


\section{RESULTADOS}

\section{Tabla 1}

Tabla de contingencia Uso de los recursos tecnológicos en los infocentros * Predisposición por parte de facilitadores para aplicar nuevos recursos tecnológicos para el aprendizaje

\begin{tabular}{lcccc} 
& \multicolumn{3}{c}{ Nuevos recursos tecnológicos para } \\
& & \multicolumn{2}{c}{ facilitar el aprendizaje } & \\
& & Casi siempre & Siempre & Total \\
\hline Utiliza los recursos tecnológicos del & Casi siempre & 2 & 3 & 5 \\
infocentros & Siempre & 2 & 66 & 68 \\
Total & & 4 & 69 & 73
\end{tabular}

Pruebas de chi-cuadrado

$\begin{array}{lccccc} & \text { Valor } & \text { gl } & \begin{array}{c}\text { Sig. asintótica } \\ \text { (bilateral) }\end{array} & \begin{array}{c}\text { Sig. exacta } \\ \text { (bilateral) }\end{array} & \begin{array}{c}\text { Sig. exacta } \\ \text { (unilateral) }\end{array} \\ \text { Chi-cuadrado de Pearson } & 12,350^{\mathrm{a}} & 1 & 0,000 & \\ \text { Corrección por continuidad } & 6,231 & 1 & 0,013 & , 022 \\ \text { Razón de verosimilitudes } & 6,234 & 1 & 0,013 & \\ \text { Estadístico exacto de Fisher } & 12,181 & 1 & 0,000 & \\ \text { Asociación lineal por lineal } & 73 & & & \\ \text { N de casos válidos } & & & \\ \text { a. } 3 \text { casillas (75.0\%) tienen una frecuencia esperada inferior a 5. La frecuencia mínima esperada es .27. } \\ \text { b. Calculado sólo para una tabla de 2x2. }\end{array}$

Fuente: Elaboración propia

Al analizar el cuestionario en la escala de Likert aplicado a los facilitadores de las provincias del Azuay y Cañar arroja como resultado de la relación entre las variables: Uso de los recursos tecnológicos en los infocentros y la predisposición por parte de facilitadores para aplicar nuevos recursos para el aprendizaje, el valor de Chi cuadrado de Pearson menor al valor crítico establecido por lo que la hipótesis en efecto es afirmativa, lo que equivale a $\mathrm{H} 1$, puesto que nos muestra la estrecha relación que existe entre la aplicación y predisposición por parte de un $80 \%$ de facilitadores para aplicar 
nuevos recursos tecnológicos.

Las variables son completamente compatibles por cuanto dependen la una de la otra tomando en cuenta que los facilitadores hacen uso de los recursos con los que cuentan en cada infocentros, pero así mismo claro está que están dispuestos a adquirir conocimientos para la aplicación de estrategias y nuevos recursos que ayudarán en la dinámica del aprendizaje de manera que en sus resultados exponen su interés por aprender y enseñar mediante el uso correcto de estos instrumentos, en tal sentido las escalas siempre y casi siempre son consideradas notoriamente en un nivel máximo que nunca y casi nunca, sabiendo que estas últimas se contradicen totalmente ya que indican que no estarían dispuestos a aplicar nuevos conocimientos y que tampoco estarían utilizando herramientas tecnológicas que tienen a su alcance.

\section{Tabla 2}

Tabla de contingencia Es indispensable promover el buen uso de las TIC * Utiliza los recursos tecnológicos del Infocentro

\begin{tabular}{|c|c|c|c|c|c|c|c|}
\hline & & & liza los recursos & ógicos del Infoc & & & \\
\hline & & & Casi siempre & Siempre & & Total & \\
\hline Es indispensable promover el buen uso & Casi & & 2 & 4 & & 6 & \\
\hline de las TIC & & & 3 & 64 & & 67 & \\
\hline Total & & & & & 68 & & 73 \\
\hline Pruebas de chi-cuadrado & & & & & & & \\
\hline & Valor & $\mathrm{gl}$ & $\begin{array}{c}\text { Sig. asintótica } \\
\text { (bilateral) }\end{array}$ & $\begin{array}{l}\text { Sig. exacta } \\
\text { (bilateral) }\end{array}$ & & $\begin{array}{l}\text { Sig. exacta } \\
\text { (unilateral) }\end{array}$ & \\
\hline Chi-cuadrado de Pearson & $7,187^{\mathrm{a}}$ & 1 & 007 & & & & \\
\hline Corrección por continuidad & 3,376 & 1 & 066 & & & & \\
\hline Razón de verosimilitudes & 4,321 & 1 & ,038 & & & & \\
\hline Estadístico exacto de Fisher & & & & 051 & & 051 & \\
\hline Asociación lineal por lineal & 7,088 & 1 & 008 & & & & \\
\hline $\mathrm{N}$ de casos válidos & 73 & & & & & & \\
\hline
\end{tabular}

Fuente: Elaboración propia 
El indicio de esta investigación respalda la idea que es indispensable promover el buen uso de las TIC y la aplicación de recursos tecnológicos necesarios para el aprendizaje, al ser analizadas mediante la prueba de Chi cuadrado se establece que estas variables se encuentran en total relación, como se puede observar en la tabla de contingencia se obtiene una frecuencia esperada inferior a 5 . Se convierte en una hipótesis de tipo afirmativa donde se demuestra que los facilitadores se encuentran en total acuerdo que es indispensable enseñar sobre el uso correcto de las TIC y su aplicación mediante recursos tecnológicos actualizados que mejoren el proceso de aprendizaje.

Al tener claras las perspectivas de los facilitadores es de suma importancia conocer si manejan aplicaciones básicas de las cuales pueden partir para emprender su enseñanza con nuevos recursos tecnológicos que se les presentará en un futuro, necesarios para acelerar y mejorar el proceso educativo para ello se presenta la información recogida en la tabla 4 que se aplica el estudio mediante el cruce de las variables: realización de presentaciones mediante powtoon y Clases impartidas mediante plataformas como: Zoom, Classroom, Facebook live, entre otras. 


\section{Tabla 3}

Tabla de contingencia Presentaciones mediante powtoon y Clases impartidas mediante plataformas como: Zoom, Classroom, Facebook live, entre otras

Clases mediante plataformas: Zoom, Classroom,

Face Life

\begin{tabular}{|c|c|c|c|c|c|c|}
\hline & & Nunca & Casi nunca & Casi siempre & Siempre & Total \\
\hline Realiza presentaciones & Nunca & 5 & 5 & 12 & 6 & 28 \\
\hline \multirow[t]{3}{*}{ con Powtoon } & Casi nunca & 0 & 6 & 8 & 3 & 17 \\
\hline & Casi siempre & 2 & 2 & 4 & 8 & 16 \\
\hline & Siempre & 0 & 0 & 1 & 11 & 12 \\
\hline Total & & 7 & 13 & 2 & 5 & 73 \\
\hline \multicolumn{7}{|l|}{ Pruebas de chi-cuadrado } \\
\hline & & \multicolumn{2}{|l|}{ Valor } & gl & \multicolumn{2}{|c|}{ Sig. asintótica (bilateral) } \\
\hline Chi-cuadrado de Pearson & & \multicolumn{2}{|c|}{$27,850^{a}$} & 9 & \multicolumn{2}{|c|}{0,001} \\
\hline Razón de verosimilitudes & & \multicolumn{2}{|l|}{30,987} & 9 & \multicolumn{2}{|c|}{0,000} \\
\hline Asociación lineal por lineal & & \multicolumn{2}{|l|}{12,499} & 1 & \multicolumn{2}{|c|}{0,000} \\
\hline N de casos válidos & & 73 & & & & \\
\hline
\end{tabular}

a. 10 casillas $(62.5 \%)$ tienen una frecuencia esperada inferior a 5. La frecuencia mínima esperada es 1.15. Fuente: Elaboración propia

La relación de las variables que fueron sometidas a la prueba de Chi cuadrado para el respectivo análisis de fiabilidad mismo que expone su resultados como $\mathrm{H} 1$, es decir una hipotesis afirmativa, pues sus datos son menores a 0,05 lo que revela una intima relación entre las variables de uso de presentaciones mediante powtoon y clases impartidas mediante plataformas como: Zoom, Classroom, Facebook live, entre otras, así se puede observar que dichos recursos tecnológicos despiertan el interés en los facilitadores para su posible aplicación dentro de estos espacios comunitarios llamados infocentros.

Una vez que han demostrado su uso, aplicación e interés por dichos recursos, se puede deducir que los facilitadores de infocentros de las provincias del Azuay y Cañar si tienen conocimientos sobre recursos tecnológicas que son utilizados para optimizar el aprendizaje que cumple su propósito mediante el uso de la tecnología, ya que pueden ser tangibles (computadora, objetos digitales)o intangibles (sistema o aplicación virtual), 
de esta manera se puede evidenciar claramente la importancia que le dan los facilitadores al uso correcto de las tecnologías y mediante sus respuesta afirmativas nos muestran que aplican los recursos necesarios para promover el aprendizaje ya que al realizar un análisis entre las tablas anteriores y sus variables demuestran con mayor fiabilidad que un $90 \%$ de facilitadores están de acuerdo con la destacada importancia que tiene el uso correcto de los recursos tecnológicos por parte de los educadores y de los educandos.

Según los estudios realizados por otros autores a cerca del tema se podría afirmar que los recuros tecnológicos son base fundamental hoy en día para mejorar el proceso de aprendizaje, por tanto requiere de mayor concentración para el desarrollo de las habilidades digitales, mismas que se pueden lograr a traves de la práctica y capacitaciones planificadas (Lattá-Arias, 2019).

Para (Castañeda, 2018), la temática de la tecnología ayuda a desarrollar el pensamiento debido al camino que recorremos y a los desafíos que se nos presentan por las situaciones actuales que nos exige la nueva era digital, así mismo en su artículo de debates nos especifica que el estudio de las TIC implica una investigación más ambiciosa en los que los autores tengan mayor protagonismo, de su parte el uso de las tecnologías de la información juegan un papel fundamental para la educación (Chirino-García \& Hernández-Corona, 2020).

De la misma manera (Jama Zambrano \& Cornejo Zambrano, 2016) corroboran en su investigación dejando en claro el nivel de importancia con respecto al desempeño del docente, pues tal como lo demuestran los resultados de su investigación el uso de recursos tecnológicos tiene un impacto positivo sobre la motivación e interés del estudiante hacia el aprendizaje. De la misma manera (Calderon \& Acuña , 2017) estipulan que los infocentros comunitarios funcionan principalmente con promotores comunitarios, que son como lo habíamos mencionado en la presente investigación los facilitadores de infocentros quienes permiten llevar a cabo la realización y reproducción del conocimiento dentro del proyecto (Perozo-Martín \& Chirinos-Martínez, 2019). 


\section{PROPUESTA}

No cabe duda de que la revolución digital nos impresiona cada vez más ya que a cambiado la manera en que las personas viven, interactuan se comunican y realizan negocios mientras experimentan un cambio profundo y rápido que tiene que ver con las herramientas tecnológicas disponibles en la actualidad por ello es necesario que los estudiantes adquieran habilidades necesarias para prepararse y afrontar todo cambio tecnológico que se nos presente en la actualidad a un futuro, por ello como lo habíamos dicho antes los infocentros que son espacios comunitarios creados con el fin de romper la brecha digital mediante capacitaciones en línea, temas que se encuentran bajo las perspectivas del PLANADI, ahora bien al tener conocimiento de estos espacios dedicados a capacitar a la ciudadanía nos preguntamos si los facilitadores dan el uso correcto a los recursos tecnológicos con los que cuentan dentro de los infocentros ya sean estos últimos tangibles o intangibles.

Para ello se recomienda a los facilitadores estar actualizados y capacitados sobre el uso correcto de los recursos tecnológicos, para ello es indispensable realizar capacitaciones continuas a los facilitadores por cuanto trabajan y actúan acorde a los instrumentos y conocimientos con los que cuentan para lo cual se contempla un proceso de tres etapas que se desarrollan a continuación.

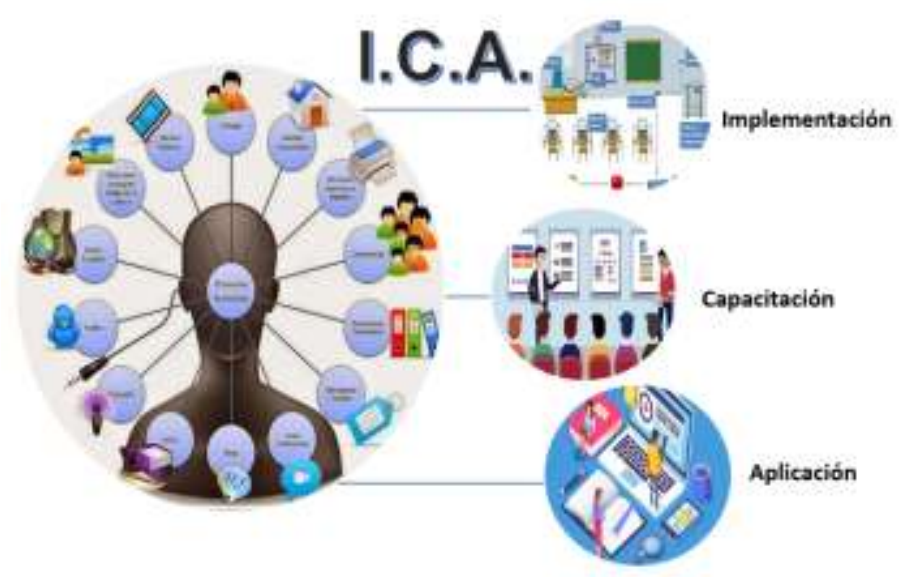

Figura 3. Proceso de implementación, capacitación y aplicación de nuevos recursos tecnológicos. Fuente: Elaboración propia. 
Debido a la predisposición que presentan los facilitadores para aprender y tomar conocimientos sobre la aplicación de nuevos recursos tecnológicos para su aplicación en infocentros se presenta un proceso basado en tres fases que son: implementación, capacitación y por último aplicación.

Implementación. En la presente etapa se da inicio a la integración de estrategias de enseñanza que podemos lograr en base a los recursos tecnológicos con los que contamos en infocentros, hoy en día para ello necesitamos estar al pendiente de las actualizaciones que se nos presentan día a día en el transcurso del avance tecnológico, para lo cual es recomendable determinar cuáles son los recursos con lo que cuentan los facilitadores.

Por cuanto los recursos intangibles podríamos modificarlos o actualizarlos en la medida de lo posible, pero los recursos tangibles dependerán de la economía en la que se encuentra cada infocentro, más sin embargo es importante destacar que encontramos un sin número de aplicaciones tecnológicas en línea con las cuales podemos trabajar e implementar a las clases y capacitaciones con los usuarios de infocentros para profundizar y dinamizar el aprendizaje de los mismos, no obstante la incorporación de las TIC en el proceso de enseñanza se debe realizar de manera gradual en los infocentros, cuidando aspectos como la infraestructura con la que cuenta este espacio educativo, así mismos con sus inmuebles esto permitiría determinar la adquisición de equipos y/o tecnología por parte de las autoridades a cargo.

Capacitación. Tal como se ha manifestado en la primera etapa sobre la importancia de la adquisición e implementación de nuevos recursos tecnológicos en infocentros, así mismo se debe prestar la debida atención a la capacitación docente o en este caso a los encargados de estos espacios comunitarios, para lo cual es recomendable contar con la debida programación de capacitaciones continuas dirigidas a los facilitadores porque si bien es cierto tienen conocimientos básicos sobre el manejo de las TIC, pero existen fallas en el proceso de enseñanza, ya sea por falta de recursos o conocimientos. 
De allí que surge la necesidad de adquirir experiencia mediante las respectivas capacitaciones que se les debe tener en cuenta mínimo cada trimestre, para que con estos conocimientos y experiencias educativas puedan transmitirlas a sus usuarios de manera fácil y didáctica logrando así conseguir un aprendizaje significativo en tal sentido que sean aplicados en el diario de vivir de cada persona y les sirva para poder lograr sus objetivos planteados.

Aplicación. Por último en la presente etapa luego de haber implementado tecnologías y conocimientos mediante las debidas capacitaciones recomendadas para los facilitadores, se encontrarán en disposición de transmitir sus conocimientos a los usuarios para lo cuál en esta fase se deberá aplicar todo lo aprendido en base a la correcta utilización de los nuevos recursos, pues de esta manera se logrará tener dentro de los infocentros a facilitadores preparados y a usuarios satisfechos que se sientan motivados para seguir adquiriendo nuevos conocimientos sobre el buen uso de las TIC y sus recursos tecnológicos, por lo tanto se espera sean puestos en práctica por los ciudadanos para que logren un aprendizaje más práctico y dinamizado.

\section{CONCLUSIONES}

Uso de los recursos tecnológicos en los infocentros y la predisposición por parte de facilitadores para aplicar nuevos recursos para el aprendizaje, el valor de Chi cuadrado de Pearson menor al valor crítico establecido por lo que la hipótesis en efecto es afirmativa, lo que equivale a $\mathrm{H} 1$, puesto que nos muestra la estrecha relación que existe entre la aplicación y predisposición por parte de un $80 \%$ de facilitadores para aplicar nuevos recursos tecnológicos.

Es indispensable promover el buen uso de las TIC y la aplicación de recursos tecnológicos necesarios para el aprendizaje, al ser analizadas mediante la prueba de Chi cuadrado se establece que estas variables se encuentran en total relación.

Se revela una intima relación entre las variables de uso de presentaciones mediante powtoon y clases impartidas mediante plataformas como: Zoom, Classroom, Facebook 
live, entre otras, así se puede observar que dichos recursos tecnológicos despiertan el interés en los facilitadores para su posible aplicación dentro de estos espacios comunitarios llamados infocentros. Se recomienda a los facilitadores estar actualizados y capacitados sobre el uso correcto de los recursos tecnológicos.

\section{FINANCIAMIENTO}

No monetario

\section{AGRADECIMIENTOS}

A la Universidad Católica de cuenca, por motivar e impulsar el desarrollo de la investigación desde la praxis educativa.

\section{REFERENCIAS}

Asamblea Nacional. (2008). Constitución De La República Del Ecuador. Asamblea Nacional(347), 107. Obtenido de https://url2.cl/tbtvU

Asamblea Nacional. (2015). Ley Orgánica de Telecomunicaciones. 88(439), 24. Obtenido de https://url2.cl/XHPJ3

Arroyo-Carrera, E., Loor-Santos, M., Mendoza-Mera, J., \& Solorzano-Zambrano, M. (2020). Gestión de aprendizaje creativo mediante la Herramienta Powtoon en estudiantes de lengua y literatura. [Creative learning management through the Powtoon Tool in language and literature students]. EPISTEME KOINONIA, 3(5), 251-267. http://dx.doi.org/10.35381/e.k.v3i5.775

Cabero Almenara, J. (2015). Reflexiones educativas sobre tecnologías de la información y comunicación TIC [Educational reflections on ICT information and communication technologies]. 1. Obtenido de https://url2.cl/G7qzs

Calderon, M. J., \& Acuña , J. (2017). Conectividad rural y cambio social: Ios Infocentros Comunitarios en el Ecuador [Rural connectivity and social change: Community Infocentres in Ecuador]. (U. C. Universidad Internacional del Ecuador, Ed.) Revista Publicando ISSN 1390-9304, 4(11), 190-207. Obtenido de https://url2.cl/SM5Xs 
Castañeda, L. (2018). Debates regarding Technology and Education: contemporary pathways and pending conversations.[Debates sobre Tecnología y Educación: Caminos contemporáneos y conversaciones pendientes]. Ried, 22(1). doi:https://doi.org/10.5944/ried.22.1.23020

Chirino-García, R., \& Hernández-Corona, J. (2020). M-learning: Estrategia para la promoción del aprendizaje electrónico móvil en instituciones de educación superior. [M-learning: Strategy for the promotion of mobile e-learning in higher education institutions]. EPISTEME KOINONIA, 3(5), 102-121. http://dx.doi.org/10.35381/e.k.v3i5.684

Delgado, A. J., Wardlow, L., Mcknight, K., \& O’Mal, K. (2015). Delgado, Educational technology: A review of the integration, resources, and effectiveness of technology in k-12 classrooms [Tecnología educativa: una revisión de la integración, recursos y efectividad de tecnologías en aulas K-12] ]. Journal of Information Technology Education: Research, 14, 398-413. doi:https://url2.cl/f2EzB

España Chavarria, C., \& Canales Garcia, A. (2013). La utilidad de las TIC para la promoción de aprendizaje en la educación superior [The use of ICT for promoting the opening of higher education.]. Teoría de la Educación. Educación y Cultura, 14(1), 189-227. Obtenido de https://url2.cl/ad5GL

Hernandez, R. M. (2017). Impacto en la educación: Retos y Perspectivas. [Impact on education: Challenges and Perspectives]. Sistema de Información Científica Redalyc., 325-347. doi:http://dx.doi.org/10.20511/pyr2017.v5n1.149

Jama Zambrano, V., \& Cornejo Zambrano, J. (2016). Los recursos tecnológicos y su influencia en el desempeño de los docentes [The technological resources and their influence on the performance of teachers]. Dominio de las Ciencias ISSN: 24778818, 2, 201,219. Obtenido de https://url2.cl/C3xvF

Lattá-Arias, C. (2019). Uso de las TIC para proyectos productivos en las instituciones educativas del Municipio Zona Bananera. Magdalena. Colombia. [Use of ICT for productive projects in educational institutions of the Municipality of the Banana Zone. Cupcake Colombia]. Revista Arbitrada Interdisciplinaria Koinonía, 4(7), 233246. http://dx.doi.org/10.35381/r.k.v4i7.202

López-Intriago, C. F., Erazo-Álvarez, J. C., Narváez-Zurita, C. I., \& Moreno, V. P. (2020). Gestión financiera basada en la creación de valor para el sector microempresarial de servicios [Financial management based on the creation of value for the services micro-business sector]. Revista Arbitrada Interdisciplinaria Koinonía, 427-454. doi:http://dx.doi.org/10.35381/r.k.v5i10.701 
Martín, F. A. (2011). La encuesta: una perspectiva general metodológica [The survey: a methodological overview]. CIS, (Vol. 35). Obtenido de https://url2.cl/1nF2n

MINTEL. (2016). Estadisticas de datos generales de Infocentros. Obtenido de https://url2.cl/ixn68

Perozo-Martín, R., \& Chirinos-Martínez, A. (2019). Incidencias de la Tecnología web 2.0 en el contexto de la gobernanza y la gobernabilidad. [Incidences of Web 2.0 Technology in the context of governance and governability]. IUSTITIA SOCIALIS, 4(6), 90-116. http://dx.doi.org/10.35381/racji.v4i6.291

Pereira-Perez, Z. (2011). Los diseños de método mixto en la investigación en educación [Mixed-method designs in educational research]. Revista electrónica educare, 15(1), 15-29. Obtenido de https://url2.cl/G9isY

Picón-Vizhñay, J., Erazo-Álvarez, J., \& Narváez-Zurita, C. (2019). Plan de expansión empresarial para la Empresa Transnexos del Grupo Industrial Graiman. [Business expansion plan for the Transnexos Company of the Graiman Industrial Group]. Revista Arbitrada Interdisciplinaria Koinonía, 274-308. doi:http://dx.doi.org/10.35381/r.k.v4i1.459

Poloni , M. (2019). Verso i musei digitali.Tecnologie digitali tra fruizione e comunicazione [Towards digital museums. Digital technologies between fruition and communication]. (U. C. Venezia, Ed.) Bachelor's thesis Università Ca' Foscari. Obtenido de https://url2.cl/si3dm

Raymond, H. (2008). Las tecnologías de la información y la comunicación para los pequeños estados insulares en desarrollo. Tesis Doctoral. Obtenido de https://url2.cl//m55S6

Serrano-de-Barrios, N. (2019). La ley de infogobierno en el contexto tecnológico de la gestión pública. [The infogovernment law in the technological context of public management]. IUSTITIA SOCIALIS, 4(7), 201-219. http://dx.doi.org/10.35381/racji.v4i7.395

Siguenza-Peñafiel, K. M., Erazo-Álvarez, J. C., \& Narváez-Zurita, C. I. (2020). Estrategias de marketing viral y el posicionamiento de marca en el sector farmacéutico [Viral marketing strategies and brand positioning in the pharmaceutical sector]. Revista Arbitrada Interdisciplinaria Koinonía 313-338. doi:http://dx.doi.org/10.35381/r.k.v5i10.697 
Vargas Espin, J., \& Parra Balza, F. D. (2018). Propuesta de marco de referencia para Teletrabajo en Ecuadr y soporte mediante tecnologías de la Información, caso de estudio infocentros Comunitarios MINTEL (Master's thesis, Quito)[Proposal of a reference framework for Telework in Ecuadr and support th]. Universidad Tecnológica Israel Escuela de Posgrados, 105. Obtenido de https://url2.cl/AnJyA

(C2020 por el autor. Este artículo es de acceso abierto y distribuido según los términos y condiciones de la licencia Creative Commons Atribución-NoComercial-Compartirlgual 4.0 Internacional (CC BY-NC-SA 4.0) (https://creativecommons.org/licenses/by-nc-sa/4.0/). 